\title{
Model Transformations with Tom
}

\author{
Jean-Christophe Bach \\ Inria, 54600, France \\ Université de Lorraine, LORIA, \\ 54500, France \\ CNRS, LORIA, 54500, France \\ jeanchristophe.bach@inria.fr \\ Pierre-Etienne Moreau \\ Inria, 54600, France \\ Université de Lorraine, LORIA, \\ 54500, France \\ CNRS, LORIA, 54500, France \\ moreau@loria.fr
}

\author{
Xavier Crégut \\ INPT-IRIT \\ Université de Toulouse \\ Toulouse. France \\ xavier.cregut@enseeiht.fr
Marc Pantel
INPT-IRIT
Université de Toulouse \\ Toulouse, France \\ marc.pantel@enseeiht.fr
}

\begin{abstract}
Model Driven Engineering (MDE) advocates the use of Model Transformations (MT) in order to automate repetitive development tasks. Many different model transformation languages have been proposed with a significant tool development cost as common language elements like expressions, statements, ... must be built from scratch for each new language development tools. The Tom language is a shallow extension of Java tailored to describe and implement transformations of tree based data-structures. A key feature of Tom allows to map any Java data-structure to tree based data abstractions that can then be accessed by powerful non-linear, associative, commutative pattern matching. In this paper, we present how this approach can be used in order to develop model transformations, in particular relying on Eclipse Modeling Framework (EMF) based metamodeling facilities. This allows to provide a transformation language at a low cost both for the development of its tools and the training of its users.
\end{abstract}

\section{Categories and Subject Descriptors}

D.2.1 [Software Engineering]: Programming TechniquesLanguages, Methodologies, Tools; D.2.11 [Software Engineering]: Software Architectures - Languages; D.3 [Programming Languages]: Miscellaneous

\section{General Terms}

Algorithms, Languages

\section{Keywords}

model transformation, language, Tom, Java, EMF, term

Here will be the copyright paragraph

Copyright 20XX XXX X-XXXXX-XX-X/XX/XX ...XXXX. structure

\section{INTRODUCTION}

One of the key success of MDE comes from its ability to abstract complex problems and to provide a standard way to model them. For a given class of problems, the specification of the modeling language is called a metamodel, and for each specific problem, the abstract representation is called a model of the problem that conforms to its metamodel. There may exist several approaches to solve a problem, each of them generally consists in presenting the abstract problem in such a way that a specialized tool or solver can be applied. One of the main activity in MDE is thus to transform problems, i.e. models expressed in a given metamodel, into problems or views expressed in another representation, i.e. in another metamodel. The transformation of models is thus at the core of MDE applications.

The notion of metamodel has been formalized by the $\mathrm{OMG}^{1}$ in the Meta Object Facility (MOF) standard ${ }^{2}$ as a subset of the UML class diagram. Intuitively, a metamodel is composed of a set of meta-classes that contain attributes and operations like usual object oriented classes. The metaclasses can be linked by inheritance, and by meta-relations, either association or composition with an associated arity. Each model must conform to such a metamodel, i.e. it is a set of elements, valued element attributes and relations between elements conforming to their meta-definitions. A model transformation is a program that takes a model as input and returns a new model, possibly conforming to the same or another metamodel.

This principle is quite simple but, as usual in software engineering, expressing sophisticated model transformations in an executable formalism can be quite complex. There are at least two main approaches to describe a model transformation:

- either a model transformation is expressed as a sequence of elementary steps that build the target model step by step (instantiating new elements, assigning attributes, creating links, etc.) using information stored

${ }^{1}$ Object Management Group, http: //www .omg.org ${ }^{2}$ http://www. omg.org/mof 
in the source model. This approach, usually called operational, is clearly imperative, as the target model is modified in each step. It can either be implemented using dedicated languages like Kermeta, QVT-Operational, ... or using reflexive libraries or generated code such as EMF 19 inside general purpose programming languages such as Java;

- or a model transformation can be defined as the relations that must exist between the source and the target models at the end of the transformation. This abstract presentation, sometimes called declarative or relational, is not directly executable, but, under some restriction, can be either translated to an operational transformation, or to a global function that takes in a step the whole source model as parameter and produces the whole target model as result (built for example with categorical pushout operators for graph rewriting based transformation languages).

Some authors 18 even consider a third architectural approach based on the introduction of an intermediate representation such as XML or any textual concrete syntax. The model transformation can then be described using a language such as XSLT or any language transformation toolset like Stratego/XT, ASF+SDF, Rascal, Spoofax, ....

Transformation language examples relying on both approaches will be presented later on in Section 5 . However, the development and the use of new languages is costly as many common features such as expressions and statements must be implemented from scratch. Furthermore, users must learn how to benefit from the new capabilities provided by the languages. The MDE community is thus balancing between the development of new expressive languages and the use of libraries in general purpose programming languages. But the tools for dedicated languages (editors, interpreters, compilers, ....) hardly ever reach the quality of general purpose languages ones; and the use of libraries that allows to manipulate models in general purpose languages either through reflexion or code generation only provide the operational approach and their use in development is quite costly. In the reflexive approach, all the type verification is done at runtime as the elements name are handled as strings. The verification activities for transformations are thus very costly. In the code generation approach, the generated code usually provides static typing but very little model querying facilities. Thus the programming cost is quite heavy as the user must either add handwritten specific queries to ease the traversal of the metamodel, or do the explicit traversal each time a data is needed.

In order to bridge the gap between these two common approaches, we consider in the following the Tom language 12 , 3], which lies halfway between them. On the one hand Tom is an extension of Java where every correct Java program is a correct Tom program. Therefore, in Tom it is possible to follow an operational approach and perform a model transformation using classical object oriented programming and EMF library. On the other hand, Tom adds declarative features to Java. An abstract term based representation can be associated to any data structure, even the most complex ones using a programmer provided, or automatically generated mapping. Sophisticated non linear associative commutative pattern matching and rule based programming can be used to define the transformations steps, without specifying in which order they are taken. Then, a specific construct called strategy is used to express the control and to specify how the transformation rules should be applied. All the constructs provided by Tom are then translated into pure Java relying on model management libraries like EMF. Thus, the end user can benefit from both classical programming, and model transformation technologies (operational and relational), without major efficiency penalty and without the additional cost of learning a completely new language. A third advantage of the proposed approach is that the language developers do not need to build a completely new language: they can rely on most existing Java tools.

In this paper, we present a technique to transform models with Tom/Java. It can be used for any model transformations as long as the metamodels can be expressed with the ECore formalism. This technique constitutes the first step towards a high-level extension of the Tom language. Section 2 introduces a practical example used in the next sections. The reader should note that the presented approach is general and is not restricted to the considered use case example; Section 3 presents the main constructs of the Tom language, tools developed to interface with EMF technology and a simple version of a model transformation; Section 4 explains how we implemented the use case in Tom and shows a generalized approach for writing models transformations with Tom; Section 5 summarizes existing model transformation languages, their advantages and drawbacks and how our proposal relates to them; Section 6 concludes and presents current and further works.

\section{FROM PROCESS MODELS TO PETRI NETS}

In the following we rely on a case study introduced by Combemale et al. in 7. This example is both simple and rich enough to illustrate problems that may occur in the general case when considering more complex model transformations. It consists in transforming process described in the SimplePDL ${ }^{3}$ formalism (Figure 1) into the Petri net formalism (Figure 2). This transformation may be used to verify properties on a process model, thanks to model-checkers based on Petri nets 6]. This verification aspect is not detailled in this paper that focuses on model transformation technologies.

\section{Meta-models}

The SimplePDL metamodel (Figure 1) defines the concept of Process composed of ProcessElements. Each process element can be either a WorkDefinition or a WorkSequence. Work definitions are the activities that must be performed during a process. A work sequence defines a dependency relationship between two work definitions. The second work definition (successor) can be started — or finished - only when the first one (predecessor) is already started — or finished - according to the value of the attribute linkType (four possible values). Finally, a work definition can be defined as a nested process (process reference), allowing the definition of hierarchal processes.

\footnotetext{
${ }^{3}$ Simple Process Description Language
} 


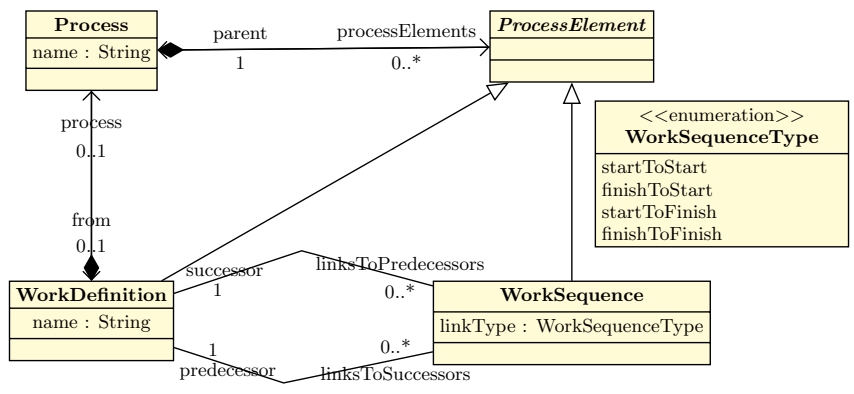

Figure 1: SimplePDL metamodel

The Petri net metamodel is shown on Figure2 A PetriNet is composed of Nodes that denote either a Place or a Transition. Nodes are linked by Arc. Arcs can be normal ones or read-arcs. An arc specifies the number of tokens (weight) consumed in the source place or produced in the target one when a transition is fired. A read-arc (second value in $A r$ cKind enumeration) only checks tokens availability without removing them. A Petri net marking is defined by the number of tokens contained in each place (marking).

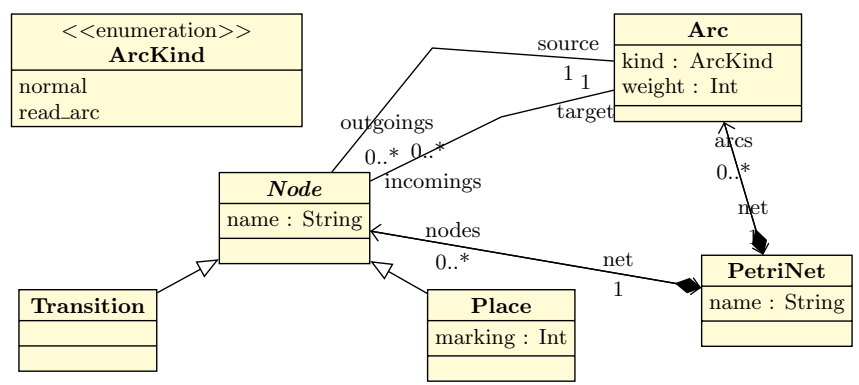

Figure 2: PetriNet metamodel

\section{Example of transformation}

The root process (Figure 3] is composed of two work definitions, $A$ and $B$ linked by a start2start work sequence, meaning that $B$ can only start after A is started. B is itself described by a process

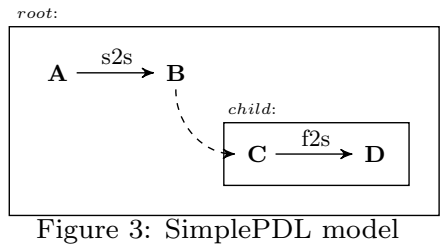

(child) composed of two activities, C and D linked by a finish2start dependency. Thus, $\mathrm{C}$ has to be finished in order to start D.

The transformation translates each process and work definition into a dedicated pre-defined Petri net template, and each work sequence into an arc. In a second step, when processes and work definitions are translated, arcs are generated to encode the synchronisation between the processes and their work definitions. A graphical representation is given below (Figure 4), where synchronisation is represented by dashed arcs, work sequences by thick green annotated arcs, places by red circles and transitions by blue squares. Details about elements composing this final Petri net are described in Section 4

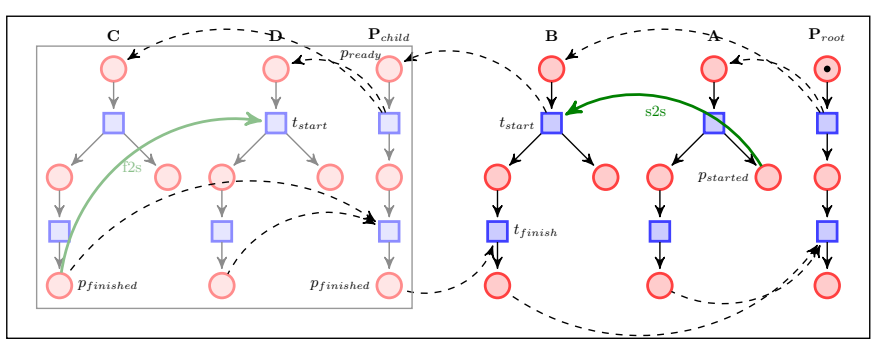

Figure 4: Complete Process described in the use case

\section{MODEL TRANSFORMATION IN JAVA WITH PATTERN-MATCHING}

To implement a model transformation such as the one sketched previously there are two main alternatives: either use a dedicated language (see Section 5), or use a general purpose language such as Java and associated model management libraries. Among the advantages of using Java we can mention efficiency, portability, and the fact that Java is a de facto industrial standard (well-known by engineers, integrated in existing processes, etc.). A main disadvantage is that Java is a low level, imperative language with respect to model transformations that is not well tailored for writing these kind of programs: there is no good support to query input models usually leading to complex code to retrieve a given piece of information and the declarative approach to model transformation cannot be expressed.

In the following we consider an extension of Java named Tom [3], whose goal is to make simpler the implementation of programs that manipulate tree-based data structures such as abstract syntax trees or XML documents for instance. The reader is invited to refer to http://tom.loria.fr for a more complete presentation.

Basically, Tom offers two new constructs: \%match, a generalized switch-case construct that allows to discriminate upon objects instead of just plain data-types such as integers, and the ' (backquote construct) which simplifies the creation of objects.

\section{Pattern matching}

The \%match statement is similar to a switch-case construct. It is composed of a list of conditions-actions: when a condition is satisfied, the corresponding action is fired. The main difference comes from the expressive power of the conditions: they are called patterns and correspond to trees that may contain variables. In the following we describe the transformation that associates a Petri net to a Process node of the input model:

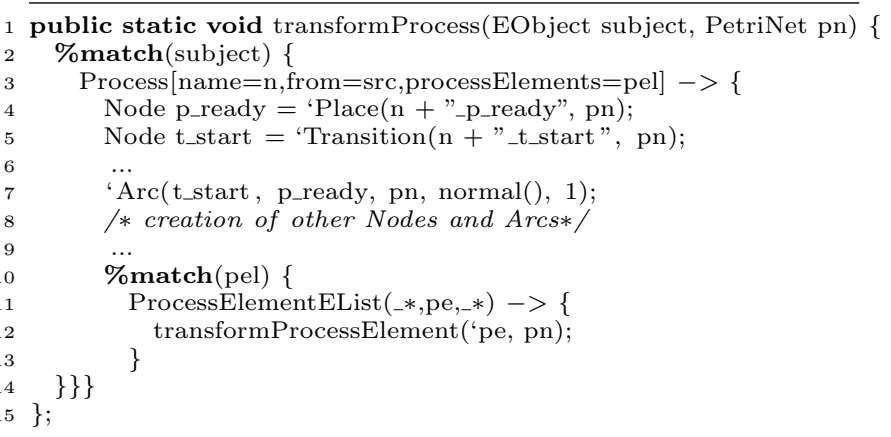


Given a subject, the pattern Process [name=n, from=src, processElements=pel] at line 3 checks that the subject corresponds to a Process node. When it is the case, the variables $\mathrm{n}$, src, and pel are initialized with the objects referenced by the attributes name, from, and processElements. In the general case, a pattern may contain nested patterns which add new constraints on the shape of sub-terms. Conjunctions (\&\&) and disjunctions (II) of patterns as well as multiple occurrences of a given variable (non linear patterns) may also be considered. The action part delimited by $\rightarrow\{\ldots\}$ may contain Java statements but also Tom statements. For instance, line 4 combines a Java assignment and a Tom backquote construct which builds a Place (i.e. a Petri net node of the output model). The 'Arc(...) expression at line 7 updates the Petri net model pn by adding an Arc between the place p_ready and the transition t_start.

The last statement (from line 10 to 12) is interesting because it shows that nested \%match constructs are allowed. It also illustrates the use of list-matching, also known as associative matching with neutral element. In this example, the pattern ProcessElementEList (_*,pe,_*) behaves like an iterator over pel: the action part is executed for each value of $p e \in$ pel.

The definition of the constructors Process and ProcessElementEList are not builtin in the language, they are derived from the metamodel given in Figure 1 relying on the EMF Java mapping. Thus, the syntax of patterns strongly depends on the considered metamodel.

\section{Algebraic views}

A \% match construct depends on two different data-structures: the subject being matched (the subject is a reference to a plain Java object) and the pattern which is expressed in an algebraic language, namely Tom. In order to compile the pattern matching constructs, the Tom compiler needs to know the relationship between the implementation of objects (Java classes), and the algebraic view (pattern sorts and constructors). For this purpose, Tom offers an algebraic view definition formalism composed of two constructs: \%typeterm and \%op. The \%typeterm, exemplified below, establishes a relation between the implementation data-type (simplepdl.Process) and the algebraic sort (Process):

1 \%typeterm Process \{ $3\}$

The following \%op constructs explains to the Tom compiler how a Java Process object can be viewed as an algebraic constructor, namely, Process:

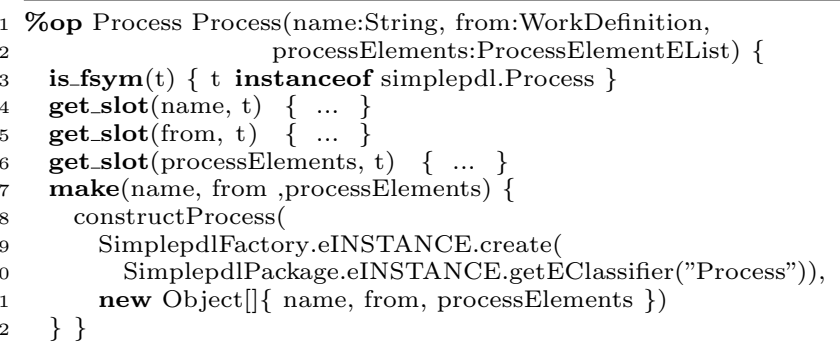

The algebraic constructor Process has three arguments, respectively of sort String, WorkDefinition, and ProcessElementElist, and its codomain is the algebraic sort Process defined above. The construct is_fsym at line 2 is used by the pattern matching algorithm to check that the current constructor (i.e. Process in this example) is the root of the algebraic representation of the Java object. It is also needed for code generation. The Java code corresponds to the implementation of this predicate. Similarly, constructs get_slot (lines 3,4 and 5) and make (line 6) define how to retrieve a given field in the data-structure, and how to build an instance of the considered constructor. This latter construct is used by the ' (backquote) construct to build terms.

For more details about the design of the Tom language, the reader is invited to refer to 12 and 3 .

\section{Towards an implementation of the transformation..}

Assuming that an algebraic view (i.e. a mapping) is defined for each element of the input and output metamodels, the considered model transformation, from SimplePDL to Petri nets, can be easily implemented using two passes over the input model: in a first pass, all WorkDefinitions are translated into Petri net templates, recursively calling transformProcess if necessary (introduced previously). In a second pass, the WorkSequences are translated into arcs according to WorkSequenceType. The resulting Petri net is a Tom term which can be used as any other term in a program. Alternately, it can be serialized by using EMF to have a standard model file usable by other tools.

This approach has a main drawback: it supposes that the transformation can be described in such a way that when building a link in the output model, the nodes it refers to have been generated in an earlier step. In the general case, this is not always possible, or this may imply an arbitrary large number of passes, which is not convenient nor efficient. A solution to this problem will be discussed and presented in Section 4.

In the following we present an automatic way to generate a mapping from a given metamodel.

\section{Generator of algebraic views}

EMF allows to generate Java code corresponding to a metamodel in a jar archive. We have designed a tool called TomEMF which uses reflection to load and inspect the classes contained in the jar archive. From theses classes, TomEMF can automatically retrieve the EClassifier (EClass and EDataType) and EStructuralFeatures in order to generate the \%typeterm and \%op constructs introduced above. For instance, when applying Tom-EMF on SimplePDL generated jar archive, the Process class produces a \% typeterm Process and a \%op Process.

When a reference indicates that an element may have multiple instances (for instance, Figure 1 shows a processElements $0 \ldots *$ relation which means that a Process can be composed of many ProcessElements), the tool generates list-matching operators such as ProcessElementEList, which are variadic, and whose matching is performed modulo associativity and neutral element.

\section{Implementation design..}

The generator could have taken an .ecore file as input instead of a .jar file. The tool implementation would have been different, since we could not have used directly Java reflection. We would have used EMF mechanism to load ECore metamodels and replaced every use of classical Java 
reflection by EMF calls. We plan to implement this generator using Tom-EMF itself.

We made the choice to generate mappings without using a full EMF reflection in order to preserve static typing, and to avoid many dynamic casts (and thus to be more efficient and to avoid potential runtime errors). A drawback is that Java files and Tom mappings have to be generated before any use (this adds an extra generation step using Eclipse).

Changes in EMF specification would not have any consequences on Tom language and the Tom compiler, but it may have an impact on the Tom-EMF mapping generator. In practice, this is not a big issue as EMF is based on the MOF standard.

\section{A GENERALIZED APPROACH FOR MO- DEL TRANSFORMATIONS USING STRA- TEGIES}

In the previous section we have shown how pattern matching capabilities offered by Tom can be used in conjunction with Java classes generated by EMF. The generation of mappings being automatic, this provides a quite simple framework that can be used to define model transformations.

However, a main drawback is the need to take care of the order in which transformation steps have to be performed, specially when the transformation has to reference elements not yet created or completed in the output model.

In the following we present a generalized two-steps approach where we separate the notion of transformation from the control of these transformations. In a first step we specify in a declarative way how each specific sub-part of the input model should be transformed. Each transformation is applied separately, leading to a model where the various sub-parts are not connected: they contain nodes, called resolve nodes, which describe the intension of being connected to another sub-part when it will become available. This approach is strongly inspired by the resolve constructs of ATL 9 and QVT 14.

In a second step, all the resolve nodes are traversed and replaced by links, to build the final output model.

\section{Generic traversal strategies}

In addition to ' (backquote) and \%match constructs, Tom offers a third construct, \%strategy, which encodes the notion of elementary transformation rule. For instance, let us consider the following snippet of code:

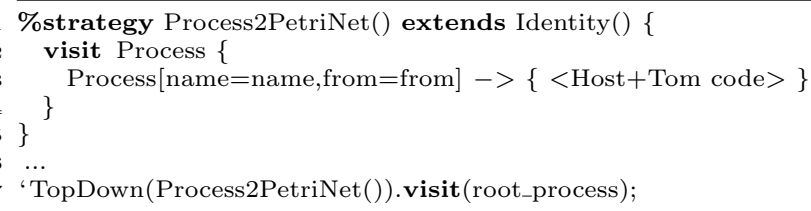

This defines an elementary transformation called Process2PetriNet whose default behavior is the Identity, meaning that no transformation occurs when the considered rule cannot be applied 4 . The visit Process constructs is a first filter which specifies the sort of objects (Process in this case) on which the rule should be applied. Then a classical Tom-rule composed of a pattern and an action is defined.

${ }^{4}$ This is in contrast to Fail which specifies that the transformation fails when the rule cannot be applied.
The main particularity of a \%strategy construct is that it is not automatically fired. Its application should be controlled by a strategy. For instance, the TopDown(Process2PetriNet ()) expression means that the rule Process2PetriNet () is applied in a top-down way on the term root_process. Tom offers several primitive strategies, such as Identity, Fail, One, All, Choice and Sequence, which can be combined, even recursively, to build more powerful strategies such as Repeat, TopDown, or Innermost for instance.

For more precise information about strategies, the reader can refer to 4 and to the dedicated pag $£^{5}$ on Tom project website.

\section{Decomposition of the transformation}

To address the issues mentioned at the beginning of this section, we propose an approach where a model transformation is specified in terms of elementary transformations which are parts of the whole transformation. Each elementary transformation being implemented by an elementary strategy. In this approach, the order of application of the transformations is not defined in the transformation itself, therefore, when an elementary transformation has to reference an element which is not yet created or completed in the output model (e.g. elements which should be created in another elementary transformation), we have to create a temporary object called resolve object, in reference to the resolveTemp construct of ATL 9 (see also resolveIn in QVT 14]). Each resolve object is stored in a two level hash-map which maps each source element we are processing (WorkDefinition in our use case) to another hash-map. This latter map connects a name (a label such as "p_ready" for example) to a target element which just has been created (the place referenced by the variable p_ready in our example).

Once each atomic transformation has been applied, the result is composed of temporary resolve objects and partial results which need to be reconnected. Thanks to the table, resolve objects can be replaced by target objects in corresponding partial results, and we obtain the final result of the transformation.

\section{Elementary transformations.}

In our use case, we consider three atomic transformations: Process2PetriNet, WorkDefinition2PetriNet, and WorkSequence2PetriNet). Each of them is implemented by a \%strategy construct.

The first one, Process2PetriNet, creates the Petri net that corresponds to the image of a Process. This Petri net is composed of three places $\left(p_{\text {ready }}, p_{\text {running }}\right.$ and $\left.p_{\text {finished }}\right)$, two transitions $\left(t_{\text {start }}\right.$ and $\left.t_{\text {finish }}\right)$ and four arcs. During the transformation, each created element (i.e. transitions and places) is stored in a hash-map (map), which is itself associated to the process being transformed ( $p$ is a variable assigned to the process which is matched). The last part of the strategy creates resolve objects: they encode the link that should be created when all parts of the model are available. In our running example, the resolve object is a reference to the transition $t_{\text {start }}$, resulting of the transformation of WorkDefinition, that will be connected via an arc to the place $p_{\text {ready }}$ of the current generated Petri net (see Figure 4 to get the "big picture"). Figure 5 shows the resulting Petri

5 http://tom.loria.fr/wiki/index.php5/ Documentation:Strategies 
net of Process2PetriNet transformation (dashed nodes are resolve nodes).

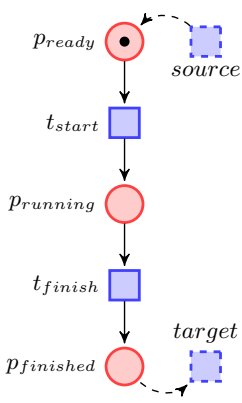

Figure 5: Petri net template for a Process

Figure 6 sketches the implementation:

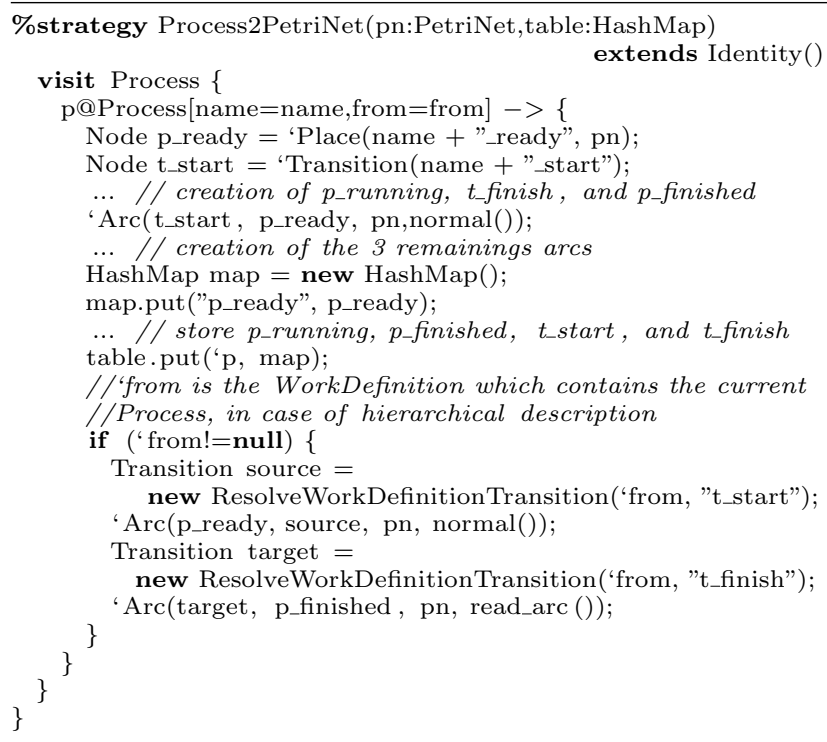

Figure 6: Process2PetriNet strategy

The two other atomic transformations implemented by WorkDefinition2PetriNet and WorkSequence2PetriNet strategies are based on the same principle, we just describe them without giving their corresponding code.

The WorkDefinition2PetriNet strategy creates all Petri net elements which define the image WorkDefinition. This Petri net is composed of four places $\left(p_{\text {ready }}, p_{\text {running }}, p_{\text {started }}\right.$ and $\left.p_{\text {finished }}\right)$, two transitions $\left(t_{\text {start }}\right.$ and $\left.t_{\text {finish }}\right)$, and five arcs (Figure 7). The only difference between a process representation and a work definition is the fact that there is an additional place $p_{\text {started }}$ after the $t_{\text {start }}$ transition. The last part of the strategy creates resolve objects representing nodes of the parent Process to which the WorkDefinition is connected.

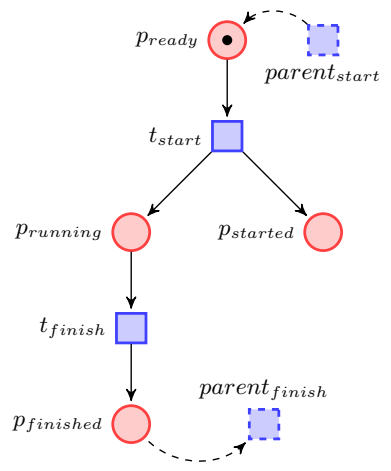

Figure 7: Petri net template for a WorkDefinition

A work sequence between two work definitions is simply represented by a read-arc between two activities (Figure 8). A read-arc controls that a transition is enabled (it checks if a token is present without removing it). Therefore, the WorkSequence2PetriNet strategy consists in creating an Arc whose source and target are both resolve nodes.

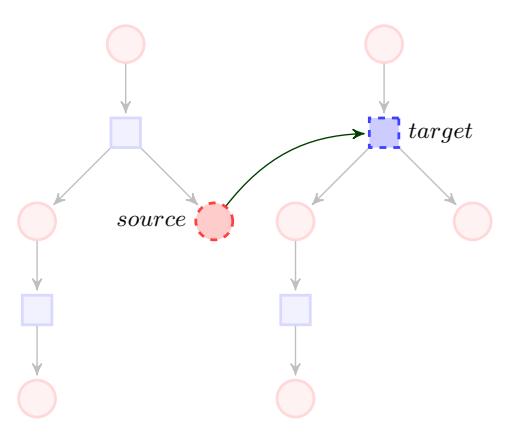

Figure 8: Petri net template a WorkSequence

\section{Controlling application of transformations.}

We have described three elementary transformations using strategies. The order in which the transformations should be applied is not encoded in the transformations themselves. Indeed, the order is not relevant and the three transformations can be applied in any order. In Figure 9 we give a possible strategy: first we transform a WorkSequence into arcs, and then we transform Process and WorkDefinition. This order has been chosen arbitrarily and could be different without any consequences.



Figure 9: Composition of builtin and custom strategies 


\section{Connecting intermediate results.}

At this stage of the presentation we have shown how a complex model transformation can be described by several independent transformations, encoded by elementary strategies. To achieve this goal we have introduced temporary resolve nodes that should be eliminated and replaced by corresponding links in the resulting model.

Since implementations of models are statically typed (thanks to EMF) we had to introduce one type of resolve object per type. For example, to implement a resolve node that corresponds to a place in a WorkDefinition, we consider the following class:

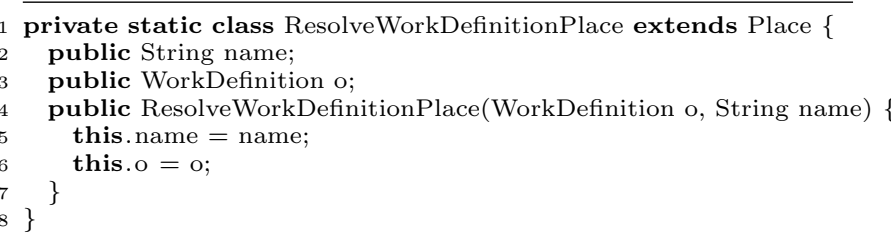

Let us now consider a simple PDL process composed by two WorkDefinition and a WorkSequence. Applying the transformer strategy leads to four unconnected Petri nets, as illustrated (Figure 10). In Figure 11 we introduce a metastrategy named Resolve, whose goal is to replace all resolve objects by the corresponding image, which are stored in the two-level hash-map. The strategy Resolve is applied in a top-down way on the resulting unconnected Petri nets that the elementary transformations produced. As a result, every Node is visited and each ResolveWorkDefinition node for instance is replaced by the node stored in the table.
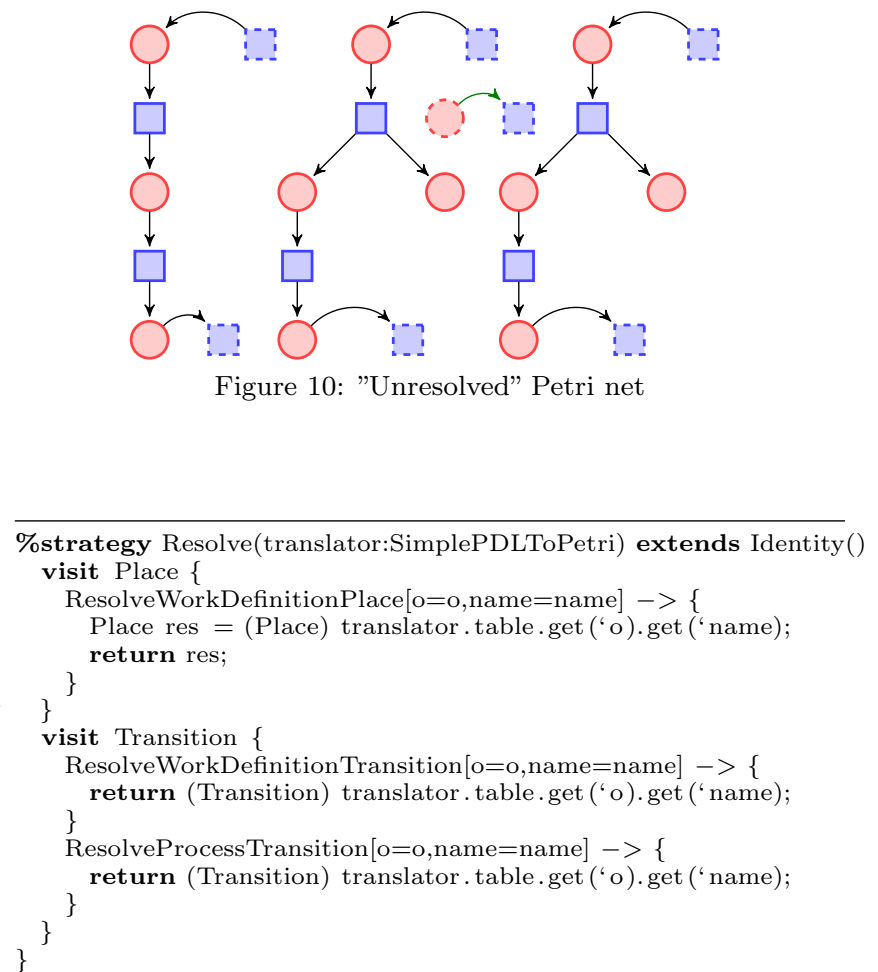

Figure 11: Resolve strategy

In the current implementation, the strategy Resolve should be manually coded by the user. We are currently experimenting an extension of the proposed approach where the Resolve strategy would be automatically inferred and generated from the elementary transformations. This is discussed in Section 6 .

\section{RELATED WORK}

This part focuses on full blown languages and experiments that have been applied on real case studies and have been available for several years either in the academic or industrial worlds.

Many languages have been designed in order to ease the writing of model transformations using model manipulation operators. These languages allow to access model contents using either graph pattern matching or object query languages usually derived from OCL. One of the differences of our approach is that instead of designing a whole new language to transform models, we use an efficient language relying on strong theoretical basis and designed to be used with general purpose languages.

The Object Management Group (OMG) has defined the Query View Transformation standard (QVT) to provide model transformation technologies for the Model Driven Architecture (MDA). Modeling languages are defined using MOF and are manipulated using OCL. The QVT standard proposes both the relational and operational approaches. The main difference is that operational ones require to describe the control as part of the transformation whereas it is handled by the execution engine for relational ones. However, the current implementations of the standard usually restrict themselves to a single one. In this paper we focus on implementation of the standard in the Eclipse world. The ATL language has been designed during the standardization process of QVT 9. It does not rely on the QVT concrete syntax but implements most of the operational and part of the relational approaches. Medini-QVT-Relationa ${ }^{6}$ implements the relational part of the standard whereas the Eclipse M2M QVT-Operationa ${ }^{7}$ implements the operational part of the standard. The relational approach consists in defining rules that map target elements and source elements. When the transformation is applied, the execution engine matches rules according to the source model and run them in the right order to avoid dangling references to not yet initialized target elements. Tom's strategies corresponds to rules and the order of rule application is defined by meta-strategies (and thus should be, at the moment, defined by the programmer).

Several languages that follow the operational approach without implementing the QVT standards are available in the EMF world. Kermet ${ }^{8}$ allows to implement methods in meta-classes 13. Model transformations are thus expressed as methods defined on the meta-classes. In order to avoid polluting the metamodels, Kermeta allows to extend metaclasses using aspect technologies. The Epsilon Transformation Language (ETL) 11 relies on a core common language that is used in many different tools in the Epsilor ${ }^{9}$ toolbox. The XTend ${ }^{10}$ language follows a similar approach. In operational approaches, programmers have full control on the

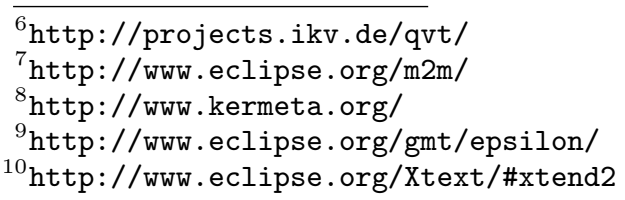


transformation but have to deal with the mapping between source and target elements, dangling references, etc. Tom's meta-strategies allow to separate the construction of parts of the target model (often described as functions in operational approach) and the sequencing of theses construction.

In the same spirit as Tom, term rewriting tools can be adapted in order to implement model transformations. Several experiments with various encodings of models have been conducted and led to some methods for using existing languages or to new toolsets. The Maude language is based on term equational rewriting systems. It provides object oriented facilities that can be used to implement metamodels and models. Its use has been experimented by several research teams 5, 16, 15] and implemented for example in the Moment ${ }^{11}$ project.

Term rewriting has been used for the last 30 years for implemented program transformations. ASF+SDF, Stratego/XT, Elan and Tom have been available for a long time and applied on real world case studies. The main issue in order to use them for model transformation is to switch from terms to graphs. The Spoofax $\sqrt{12}$ toolset is based on Stratego/XT 10, 8].

Graph rewriting for model transformations has been experimented in the last 20 years 20,17 ] using various categorical encodings like single and double pushout and specification formalism like graph types or triple graph grammars. Many tools have been implemented. The following ones are currently available in the Eclipse world. The Moflor ${ }^{13}$ toolset relies on triple graph grammar in order to implement model transformations 1 . The Henshir ${ }^{14}$ project is the followup of many experiments 2] in Eclipse based on the AGG toolset. The main issue of graph rewriting is that it relies on quite costly synthesis technologies in order to build the transformation function from the elementary graph rewriting rules and the scalability of the categorical technologies is still to be experimented.

In the same spirit as the Tom shallow language extension approach, MPS the Meta Programming System, relies on lightweight language extensions that are translated to the core language, currently Java. MPS provides an integrated tool for the definition of languages extensions. It could thus be used for Tom implementation. But, it does not provide currently model transformation extension to Java. Tom also relies on state of the art term rewriting technologies and especially efficient term management and pattern matching.

\section{CONCLUSION AND FUTURE WORK}

In this paper we have presented the Tom programming language and we have showed how pattern matching and algebraic views can be used to encode model transformations in a more abstract way than in pure Java, using EMF.

In a second part, we have introduced the notion of strategy, and we have showed how they can be used to encode elementary transformations, where the notion of scheduling is no longer part of the transformation itself.

To achieve this result, we have introduced intermediate

${ }_{11}^{1}$ http://www.cs.le.ac.uk/people/aboronat/tools/
moment2/
${ }^{12}$ http://strategoxt.org/Spoofax
${ }^{13}$ http://www.moflon.org/
${ }^{14}$ http://www.eclipse.org/modeling/emft/henshin/
${ }^{15}$ http://www.jetbrains.com/mps/

QVT derived resolve objects and a two-level hash-map that stores them. Then, we have showed how they can be replaced by links of the model, using an elegant TopDown(Resolve()) meta-strategy.

What we have presented is a first step towards a high-level language integrated into Java. The method we presented can be used to specify any model transformation between any two different models. Our next objective is to introduce a higher-level construct \%transformation which automates the generation of most of the code presented previously: elementary strategies, resolve objects classes, and the Resolve meta-strategy can be automatically generated.

This new construct will be composed of model transformation rules, and each rule would be compiled as a strategy. A developer will only have to write the transformation itself without having to take care of resolve objects, the two-level hash-map, as well as the Resolve strategy. All the needed information will be encoded in the transformation, using a domain specific syntax dedicated to the transformation of models. The goal is to simplify the writing of models transformations in the Java world.

The second part of incoming work is the extension of the strategy language to offer the possibility to create parametrized strategies. Parameter could be the type of link the transformation developer wants to follow. It would make it more flexible.

A third part of future work will be the extension of the mapping generator itself: for the moment, it is EMF-based, but we could generalize it to handle other technologies.

Once Tom language will be extended, we will be able to express complex transformations in Java in an easy way. Then we will be able to build complete execution traces to verify models transformations. With those traces, it will be possible to reconstruct the transformations chain to point a potential problem (for example, given by the results of a model-checker).

\section{REFERENCES}

[1] C. Amelunxen, A. Königs, T. Rötschke, and A. Schürr. Moflon: A standard-compliant metamodeling framework with graph transformations. In A. Rensink and J. Warmer, editors, ECMDA-FA, volume 4066 of Lecture Notes in Computer Science, pages 361-375. Springer, 2006.

[2] T. Arendt, E. Biermann, S. Jurack, C. Krause, and G. Taentzer. Henshin: Advanced concepts and tools for in-place emf model transformations. In D. C. Petriu, N. Rouquette, and $\varnothing$. Haugen, editors, MoDELS (1), volume 6394 of Lecture Notes in Computer Science, pages 121-135. Springer, 2010.

[3] E. Balland, P. Brauner, R. Kopetz, P.-E. Moreau, and A. Reilles. Tom: piggybacking rewriting on java. In Proceedings of the 18th international conference on Term rewriting and applications, RTA'07, pages 36-47, Berlin, Heidelberg, 2007. Springer-Verlag.

[4] E. Balland, P.-E. Moreau, and A. Reilles. Rewriting strategies in java. Electr. Notes Theor. Comput. Sci., 219:97-111, 2008.

[5] A. Boronat and J. Meseguer. Moment2: Emf model transformations in maude. In A. Vallecillo and G. Sagardui, editors, JISBD, pages 178-179, 2009.

[6] B. Combemale, X. Crégut, P.-L. Garoche, and $\mathrm{X}$. Thirioux. Essay on Semantics Definition in MDE. 
An Instrumented Approach for Model Verification. Journal of Software, 4(6), 2009.

[7] B. Combemale, P.-L. Garoche, X. Crégut, X. Thirioux, and F. Vernadat. Towards a Formal Verification of Process Model's Properties SimplePDL and TOCL Case Study. In ICEIS (3), pages 80-89, 2007.

[8] Z. Hemel, L. C. L. Kats, D. M. Groenewegen, and E. Visser. Code generation by model transformation: a case study in transformation modularity. Software and System Modeling, 9(3):375-402, 2010.

[9] F. Jouault, F. Allilaire, J. Bézivin, and I. Kurtev. Atl: A model transformation tool. Science of Computer Programming, 72(1-2):31-39, June 2008.

[10] L. C. L. Kats and E. Visser. The spoofax language workbench: rules for declarative specification of languages and ides. In W. R. Cook, S. Clarke, and M. C. Rinard, editors, OOPSLA, pages 444-463. ACM, 2010.

[11] D. S. Kolovos, R. F. Paige, and F. Polack. The epsilon transformation language. In A. Vallecillo, J. Gray, and A. Pierantonio, editors, ICMT, volume 5063 of Lecture Notes in Computer Science, pages 46-60. Springer, 2008.

[12] P.-E. Moreau, C. Ringeissen, and M. Vittek. A pattern matching compiler for multiple target languages. In G. Hedin, editor, Compiler Construction, volume 2622 of Lecture Notes in Computer Science, pages 61-76. Springer Berlin / Heidelberg, 2003.

[13] P.-A. Muller, F. Fleurey, and J.-M. Jézéquel. Weaving executability into object-oriented meta-languages. In L. C. Briand and C. Williams, editors, MoDELS, volume 3713 of Lecture Notes in Computer Science, pages 264-278. Springer, 2005.

[14] Object Management Group, Inc. Meta Object Facility (MOF) 2.0 Query/View/Transformation (QVT) Specification, version 1.0, Apr. 2008.

[15] J. R. Romero, J. E. Rivera, F. Durán, and A. Vallecillo. Formal and tool support for model driven engineering with maude. Journal of Object Technology, 6(9):187-207, 2007.

[16] V. Rusu. Embedding domain-specific modelling languages in maude specifications. ACM SIGSOFT Software Engineering Notes, 36(1):1-8, 2011.

[17] A. Schürr and F. Klar. 15 years of triple graph grammars. In H. Ehrig, R. Heckel, G. Rozenberg, and G. Taentzer, editors, ICGT, volume 5214 of Lecture Notes in Computer Science, pages 411-425. Springer, 2008.

[18] S. Sendall and W. Kozaczynski. Model transformation: the heart and soul of model-driven software development. Software, IEEE, 20(5):42-45, 2003.

[19] D. Steinberg, F. Budinsky, M. Paternostro, and E. Merks. EMF: Eclipse Modeling Framework 2.0. Addison-Wesley Professional, 2nd edition, 2009.

[20] G. Taentzer. What algebraic graph transformations can do for model transformations. ECEASST, 30, 2010 . 